\title{
Characterization of a rat model of metastatic prostate cancer bone pain
}

\author{
Paolo Donato De Ciantis' \\ Kiran Yashpal² \\ James Henry ${ }^{3}$ \\ Gurmit Singh' \\ 'Department of Pathology and \\ Molecular Pathology, ${ }^{2}$ Pain Research \\ Laboratories, ${ }^{3}$ Department \\ of Psychiatry and Behavioral \\ Neurosciences, McMaster University, \\ Hamilton, Ontario, Canada
}

This article was published in the following Dove Press journal: Journal of Pain Research

3 November 2010

Number of times this article has been viewed
Purpose: The objectives of this study were to establish and characterize a novel animal model of metastatic prostate cancer-induced bone pain.

Methods: Copenhagen rats were injected with $10^{6}$ MATLyLu (MLL) prostate cancer cells or phosphate-buffered saline by per cutaneous intra femoral injections into the right hind leg distal epiphysis. Over 13 days, rats progressively developed a tumor within the distal femoral epiphysis. On days 3, 7, 10, and 13 post injection, rats were subjected to the incapacitance and Randall-Selitto behavioral tests as they are believed to be indirect reflections of tumor induced pain. Ipsilateral hind limbs were subjected to X-ray and computed tomography (CT) scans and histological sections were stained with hematoxylin and eosin (H\&E).

Results: Intra femoral injections of MLL cells resulted in the progressive development of a tumor leading to bone destruction and nociceptive behaviors. Tumor development resulted in the redistribution of weight to the contralateral hind leg and significantly reduced the paw withdrawal threshold of the ipsilateral hind paw as observed via the incapacitance and Randall-Selitto tests, respectively. X-ray and computed tomography scans along with H\&E stains indicated tumorassociated structural damage to the distal femur. This model was challenged with administration of meloxicam. Compared with vehicle-injected controls, the meloxicam-treated rats displayed smaller nociceptive responses as observed with the incapacitance and Randall-Selitto tests, suggesting that meloxicam was effective in reducing the pain-related symptoms displayed by model animals and that the model behaved in a predictable way to cyclooxygenase- 2 treatment.

Conclusions: This model is unique from other bone cancer models in that it is a comprehensive model utilizing a competent immune system with a syngeneic tumor. The model establishes a tool that will be useful to investigate mechanisms of cancer pain that are induced by cancer cells.

Keywords: tumor, nociception, behavior, meloxicam

\section{Introduction}

The skeleton is a preferred site for the metastasis of malignant tumors, particularly for prostate cancer. ${ }^{1-5}$ Prostate cancer remains a serious health concern for males as the lifetime risk is $19.8 \%$, and it is the second leading cause of cancer-related deaths in men. ${ }^{6}$ The majority of males dying from prostate cancer have some form of skeletal involvement at death. ${ }^{7,8}$ Furthermore, skeletal metastases have been identified in approximately $70 \%$ of patients with breast or prostate cancer, while some form of skeletal metastasis has been identified in more than $90 \%$ of patients who die from breast or prostate cancer. ${ }^{8-9}$

Patients suffering from bone tumors frequently experience a reduced quality of life due to a variety of factors that include hypercalcemia, bone fractures, spinal
Correspondence: Gurmit Singh Pathology and Molecular Medicine, McMaster University,

699 Concession Street, Hamilton, Ontario L8V5C2, Canada

Tel + I 90538797 I I ext 67004

Fax +l 9055756330

Email gurmit.singh@jcc.hhsc.ca 
cord compression, and severe bone pain.,.$^{3,4,6,9-17}$ The most common symptom experienced by patients with bone tumors is pain. ${ }^{3,11,12,14,18,19}$ Due to the incidence, the severity, and the impact of metastatic bone cancer pain, a project was initiated to develop a novel animal model. The novel bone cancer animal model was induced by the introduction of prostate cancer cells into a hind leg distal femoral epiphysis providing an experimental model with which to investigate mechanisms of bone cancer pain.

While the severity and evolution of bone pain experienced by patients varies, cancer-induced bone pain (CIBP) is typically divided into two categories: ongoing pain and breakthrough or incident pain. ${ }^{3,20-22}$ Typically presenting first, ongoing pain is described as a constant dull or throbbing pain that increases in intensity over time. Ongoing pain can be intensified with the active use of skeletal segments affected by tumor. ${ }^{3}$ With the progression of bone cancer, breakthrough or incident pain begins to present itself. Breakthrough or incident pain is characterized as periodic exacerbations of bone pain. ${ }^{3,20-25}$ Breakthrough pain can arise spontaneously and be unrelated to movement or can be associated with the movement of tumor affected skeletal portions. ${ }^{13,20-22}$

In 1961, Dunning observed the appearance of an adenocarcinoma in the prostate of a male Copenhagen rat. ${ }^{26}$ The Dunning R-3327 adenocarcinoma is a spontaneously developed prostate tumor derived from a male Copenhagen rat. $^{27,28}$ The Dunning R-3327 tumor line has a doubling time of 15-20 days and is androgen sensitive. ${ }^{27}$ Continuous subcutaneous passage of the R-3327 tumor led to the production of the rapidly growing (doubling time of approximately 2.2 days) R-3327 AT (anaplastic tumor) tumor cell line which is androgen insensitive with a low metastatic potential. ${ }^{26,27,29,30}$ At the 60th passage, the R-3327 AT tumor cell line displayed a high metastatic potential and increased growth rate (doubling time of approximately 1.5 days) and was renamed the MAT (metastatic-AT) tumor. ${ }^{27}$ The new MAT tumor cell line reproducibly resulted in the metastatic development of tumors at the lymph nodes and within the lungs. ${ }^{27,31}$ As such, the tumor cell line was dubbed the MATLyLu or "metastatic anaplastic tumor capable of spreading to the lymph nodes and lungs" cell line. ${ }^{27}$ MATLyLu (MLL) cells have an in vitro doubling time of 19.7 hours. Also, MLL cells are characterized as spindle or polygonal shaped and possess large nuclei. ${ }^{31}$

Meloxicam is a nonsteroidal anti-inflammatory drug that is produced from enolic acid. ${ }^{32}$ The chemical name of meloxicam is 4-hydroxy-2-methyl-N-(5-methyl-2-thiazolyl)-2H-1,2benzo-thiazine-3-carboxamide-1,1-dioxide. ${ }^{33}$ Meloxicam preferentially inhibits the activity of COX-2 over COX-1, particularly at lower therapeutic doses. ${ }^{34,35}$ This preferential inhibition of COX-2 is likely due to the unique structure of meloxicam. In studies where the $4^{\prime}$ isomer of meloxicam is modified, COX-2 specificity is lost. ${ }^{36}$ Meloxicam provides analgesia through the inhibition of COX-2 enzymatic activity ${ }^{32,37}$ Furthermore, meloxicam has demonstrated inhibitory effects on cancer growth as seen in studies on colorectal and nonsmall cell lung cancer cells. ${ }^{38,39}$ However, to date, no studies have been performed to evaluate the effects of meloxicam on CIBP. As such, this study is novel in its charge to evaluate the effects of meloxicam in a model of CIBP.

Pre-existing tumors in the breast, prostate, and lung commonly metastasize to axial skeletal components, including the vertebrae. Vertebral bodies are comprised mainly of trabecular bone surrounded by a cortical bone shell. The distal femoral epiphysis, an appendicular skeletal component, consists of trabecular bone surrounded by a dense cortical bone shell. Within the animal model utilized in this project, the distal femoral epiphysis acts as a model for the metastasis of a primary tumor (prostate) to the vertebrae. As a result, the nociceptive consequences of prostate cancer metastasis can be studied. Moreover, the specific placement of cancer cells within the rat femur allows the tumor to develop over a longer period than would be possible in the confined rat vertebrae. Due to its proximity to the spinal cord, even small tumor growth within the vertebrae of the rat could have immediate detrimental consequences. As such, the development of tumors within the vertebrae of rats would likely require euthanasia prior to or immediately following the commencement of behavioral tests. However, the placement of tumor cells within the femur would allow tumor growth over a longer period of time facilitating data collection. It was hypothesized that injection of MLL cells into the distal femoral epiphysis of a rat results in the production of a bone tumor that leads to the generation of nociceptive behaviors.

\section{Materials and methods MLL cell maintenance}

Based on confluence, flasks containing cultured MLL cells were split every third to fourth day. Fresh media (RPMI 1640; Sigma-Aldrich Canada Ltd, Oakville, ON, Canada) was warmed for approximately 30 minutes at $36^{\circ} \mathrm{C}$ prior to use. Frozen trypsin (Gibco 15400, 0./E5\% trypsinDTA; Invitrogen Canada Inc, Burlington, ON, Canada) was also warmed for approximately 30 minutes at $36^{\circ} \mathrm{C}$ prior to use. Approximately $2 \mathrm{~mL}$ of trypsin was flushed over the cell monolayer. Fresh media was then added to incubating flasks 
to inactivate trypsin. Cell and media mix was then transferred via sterile pipette to new sterile T-75 flasks and returned to the cell incubator.

\section{Model induction-MLL cell preparation}

Media from growth flasks was removed via sterile pipette prior to addition of $2 \mathrm{~mL}$ of trypsin. Growth flasks were then gently rocked and left at room temperature for approximately 3-5 minutes. Fresh media $(8 \mathrm{~mL})$ was then flushed over the growth surface in order to inactivate trypsin. For one flask, $10 \mathrm{~mL}$ of the cell suspension was placed into a $50 \mathrm{~mL}$ conical tube topped up to $20 \mathrm{~mL}$ with fresh media. After mixing, $50 \mu \mathrm{L}$ of the cell suspension was removed in order to facilitate cell counting. The cell suspension was then centrifuged at $1000 \mathrm{rpm}$ for 5 minutes to induce pellet formation. Following centrifugation, the supernatant fraction was carefully removed via sterile pipette. A predetermined volume of phosphate-buffered saline (PBS; vehicle) was then added to the pellet to allow for a cell concentration of $1.0 \times 10^{7}$ cells $/ \mathrm{mL}$. MLL cells were then resuspended within PBS with use of a sterile pipette. For vehicle injections, fresh PBS was transferred to a sterile flask with use of a sterile pipette and sealed within a biological safety cabinet.

\section{Animals}

All experiments were performed on male Copenhagen rats (Harlan Laboratories Inc., Indianapolis, IN) weighing 225-275 g. All rats were housed in a controlled environment with a 12-hour rotating light and dark cycle. Food and water were provided ad libitum. Experiments were reviewed and approved by the Animal Research Ethics Board at McMaster University. All animals were cared for and used in accordance with The Care and Use of Experimental Animals, Volumes 1 and 2, of the Canadian Council on Animal Care.

\section{Induction of model}

Anesthesia of rats was achieved with gaseous isoflurane and oxygen mixture. The right hind leg (ipsilateral) femur/tibia joint was flexed from $45^{\circ}$ to $55^{\circ}$ allowing distal patellar movement. A 25 ga needle was aligned parallel to the long axis of the ipsilateral femur, between both the medial and lateral condyles. Following insertion through cutaneous tissue, the needle was rotated manually to induce cavity formation within the epiphysis. MLL cells $\left(1.0 \times 10^{6}\right)$ suspended in $0.10 \mathrm{~mL}$ PBS were then injected into the bone for tumor induction. Control rats received a $0.10 \mathrm{~mL}$ PBS injection into the epiphysis. Volume of injected material was minimized to ensure that it remained within the penetrated epiphysis.
Accessory surgical instrument, procedures, and materials were avoided in order to minimize damage to tissue and ensure observed nociceptive behaviors were due to tumor growth within the epiphysis.

\section{Drug administration}

Meloxicam (meloxicam sodium salt hydrate; Sigma-Aldrich Canada Ltd) was dissolved in 5\% methyl cellulose (M70140500MG powder; Sigma-Aldrich Canada Ltd,) prepared on testing days prior to commencement of behavioral tests. Meloxicam was administered as $5,2.5$, or $1 \mathrm{mg} / \mathrm{kg}$ intraperitoneal injection. In order to allow for meloxicam to be metabolized and take effect, behavioral tests began 45 minutes following meloxicam or PBS injection.

\section{Characterization of model}

Baseline (BL) readings were recorded from all rats from the incapacitance and Randall-Selitto tests prior to model induction. Results from the tests were then recorded from all rats on days $3,7,10$, and 13 post-induction. On experimental days, all rats first underwent the incapacitance test followed then by the Randall-Selitto test. It was determined experimentally that 13 days following model induction, rats experienced serious and unacceptable detrimental effects as a result of tumor growth. These effects were observed in behaviors that included, but were not limited to, previously unobserved defensive posture in the presence of the experimenter, vocalization upon palpation and severely reduced ambulation. As such, due to ethical considerations, it was determined that all experiments would be terminated 13 days following model induction.

A Dual Channel Weight Averager, Incapacitance Tester (Linton Instrumentation, Norfolk, UK), was utilized to measure differential hind leg paw weight distribution. Prior to data collection, rats were placed in a clear plastic holder. Below the level of the holder and under the rat, two force transducer pads measured the weight placed upon each hind leg. The forelimbs of rats were rested halfway upon the leading edge of the plastic holder. An individual recording comprised of the average weight (in grams) placed on each hind paw that was automatically recorded over a preset period of 3 seconds for each recording. On each testing day, three individual recordings were obtained per rat.

An IITC Model 2500 Randall-Selitto Paw Pressure Meter (IITC Life Science Inc., Woodland Hills, CA) was utilized for data collection in order to examine skin hyperalgesia. Due to the diameter of the rat femur (which progressively increased following tumor development), rat paws were tested. Rats 
were lightly restrained within a cloth with the ipsilateral hind leg exposed and free to move. This hind paw was placed on the paw pressure clamp while the plinth was depressed onto the anterior surface of the paw. The minimum force (in grams) applied to the paw prior to its voluntary withdrawal and subsequent dislodging from the pressure clamp was automatically recorded. On each testing day, three individual recordings were obtained per rat taken 2 minutes apart.

On all testing days prior to the commencement of behavioral tests, the weight of all rats was measured and recorded.

\section{Histology}

Upon euthanasia, both the ipsilateral and contralateral hind legs of rats were immediately dissected and shed of cutaneous tissue and muscle. Following dissection, all bone samples were immediately fixed in a $10 \%$ formalin solution in PBS

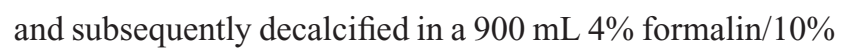
ethylenediaminetetraacetic acid (EDTA) solution. Samples remained within the solution for 4 weeks with the replacement of fresh solution occurring every third day.

Once decalcification was complete, samples were further processed in paraffin wax. Sections were placed on ice cubes to cool over a period of 10-15 minutes. When cool, sections were fixed to a microtome (Reichert-Jung 2040 Microtome; Reichert Inc., Depew, NY) and $3 \mu \mathrm{m}$ sections of tissue were produced. Sections were then placed onto a preheated water bath. Tissue was fixed onto the surface of appropriately labeled glass slides and allowed to dry overnight.

Glass slides possessing sectioned tissue were placed into an oven at $45^{\circ} \mathrm{C}$ for $20-25$ minutes prior to hematoxylin and eosin (H\&E) staining. Slides were subsequently removed from the oven and allowed to cool for 10-15 minutes. Once cool, slides were placed within a slide holder and washed until clear in three consecutive changes of xylene for 5 minutes each. The slides were then washed until clear within $100 \%$ ethanol, $100 \%$ ethanol, $70 \%$ ethanol, and $50 \%$ ethanol sequentially. Following ethanol washes, slides were immersed within water then treated in hematoxylin (Gill Number 3, GHS332-1 L; Sigma-Aldrich Canada Ltd), diluted with water to a ratio of 1:2 for 3 minutes to induce staining, followed by immersion in water. Slides were then immersed within alkaline lithium carbonate for 10 seconds to change the color of the hematoxylin stain to blue. Lithium carbonate treatment was followed by a water wash. Slides were then immersed for 45 seconds in eosin solution. Eosin (diluted $1: 3$ in $80 \%$ ethanol) treatment was then followed with two washes until clear of $96 \%$ ethanol, three changes of $100 \%$ ethanol, and two changes of xylene. Cover slips were then mounted on top of slides with Permount (SP15-100 Toluene Solution; Fisher Scientific Company, Toronto, ON, Canada) and allowed to dry overnight.

\section{X-ray radiographs}

Following the completion of all behavior tests, rats were euthanized and hind limbs were immediately dissected and placed within $10 \%$ formalin. High-resolution radiographic scans of dissected rat femurs were acquired with a Faxitron X-ray MX-20 system (Faxitron, Lincolnshire, IL) on Kodak MIN-R 2000 Mammography Film (MIN-R 2000 Mammography Film, Kodak, Rochester, NY).

\section{CT scans}

Following the completion of all behavior tests, rats were euthanized, and hind limbs were immediately dissected and placed in $10 \%$ formalin. Computed tomography (CT) scans were obtained with a GammaMI Gamma Medica X-SPECT Dedicated Animal Imaging unit (Gamma Medica-Ideas Ltd., London, ON, Canada). Data for each sample were reconstructed with Cobra_EXXIM software (Exxim Computing Corporation, Pleasanton, CA).

\section{Statistical analysis}

Behavior data are presented as the mean \pm standard deviation (SD). Differences in bodyweight data are presented as differences from $B L$ values \pm SD. Statistically significant differences between groups were determined with two-way analysis of variance (ANOVA) followed by Bonferroni post hoc analysis. A two-tailed $P$-value of less than 0.05 was considered significant.

\section{Results H\&E stain}

The H\&E-stained sections of the ipsilateral distal femur from PBS-injected rats indicated the presence of healthy marrow within the epiphysis and diaphysis. Healthy trabecular bone was also visible in sections from the PBS-injected rats. Sections of the ipsilateral distal femur from MLL cell injected rats indicated tumor replacement of marrow within the epiphysis (Figure 1). Also visible within the epiphysis of MLL cell injected rats was eroded trabecular bone (Figure 1). As in the sections from PBS-injected rats, those from MLLinjected rats revealed the presence of healthy marrow within the femoral diaphysis. 


\section{X-ray radiographs}

Comparisons were made of radiographs of the ipsilateral hind legs from PBS and MLL cell injected rats. Representative radiographs are illustrated in Figure 2. PBS-injected rats exhibited normal bone density and structure in the ipsilateral hind leg. Clinical features of structural changes visible in the radiographs of MLL cell injected ipsilateral hind leg illustrated in Figure 2 were not observed in the ipsilateral hind leg of vehicle-injected rats.

\section{CT scans}

Comparisons were made of CT scans of the ipsilateral hind legs from PBS and MLL cell injected rats. CT scans from

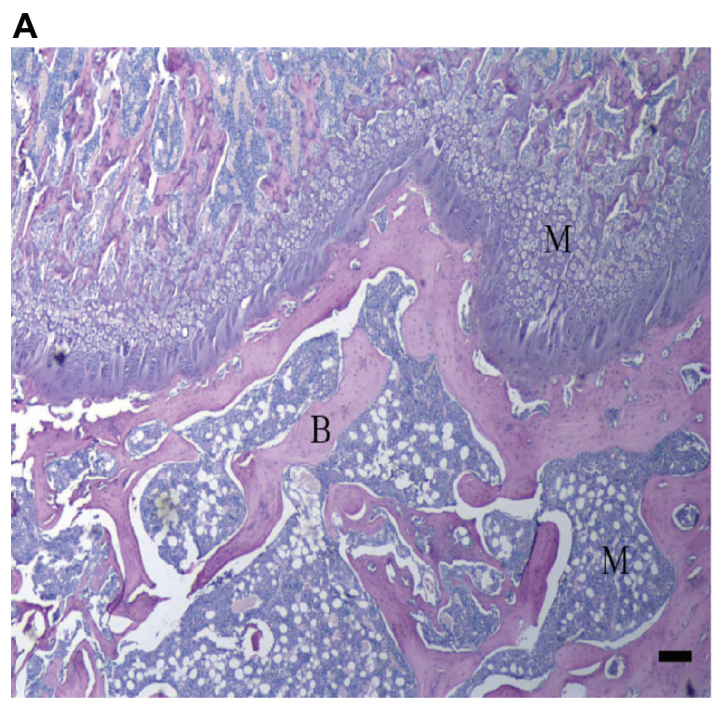

B

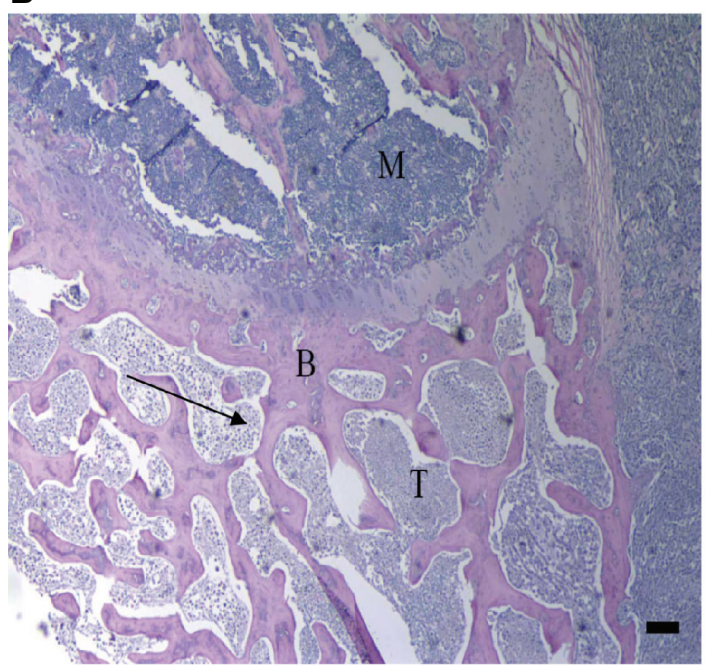

Figure I Hematoxylin and eosin (H\&E) stains of ipsilateral distal femur. Histological sections of the ipsilateral femoral epiphysis from vehicle (A) and MLL (MATLyLu) cell injected (B) rats. Visible in the figure is eroded trabecular bone (arrow). Sections were stained with H\&E. M, marrow, B, bone, T, tumor. Bar represents $100 \mu \mathrm{m}$.
PBS-injected rats indicated healthy ipsilateral hind legs with a lack of bone degradation in the distal epiphysis. Scans from MLL cell injected rats indicated extensive osteolytic damage to the distal epiphysis of the ipsilateral hind leg (Figure 3).

\section{Difference in bodyweight}

Figure 4 shows the change in bodyweight from $\mathrm{BL}$ of rats following PBS or MLL cell injection. The difference in this weight change in the MLL group was significantly different from that in the PBS injected group on days 10 and 13 $(P<0.001)$. Weight gain in the MLL cell injected group plateaued between days 7 and 10 with a loss in weight between days 10 and 13. PBS-injected rats exhibited normal weight gain over time associated with normal growth. Data are displayed as mean $\pm \mathrm{SD}$.

\section{Incapacitance test}

Prior to model induction, both control and model rats were observed to place weight evenly on both hind legs, thus displaying a differential weight distribution of approximately zero when placed in the chamber. Figure 5A shows changes in hind limb weight distribution (contralateral-ipsilateral) following PBS or MLL cell injection. The MLL cell injected group was significantly different from the PBS-injected group on days 7,10 , and $13(P<0.001)$. The MLL cell-injected rats preferentially placed weight on the contralateral hind leg after day 3. PBS injected rats maintained BL level of weight distribution.

\section{Randall-Selitto test}

Prior to model induction, both groups of rats voluntarily withdrew their ipsilateral paw at approximately 130 g. Figure 5B depicts change in ipsilateral paw withdrawal threshold from the Randall-Selitto pressure clamp following PBS or MLL cell injection. The MLL cell-injected group was significantly different from PBS-injected group on days 7, 10, and 13 $(P<0.001)$. PBS-injected group maintained BL level of withdrawal threshold.

\section{Effect of meloxicam in the incapacitance test}

Figure 6A shows hind limb weight distribution (contralateralipsilateral) following MLL cell injection at different doses of meloxicam. The vehicle group was given a 5\% methyl cellulose solution. The $5.0 \mathrm{mg} / \mathrm{kg}$ group was significantly different from the $2.5 \mathrm{mg} / \mathrm{kg}$ and $1.0 \mathrm{mg} / \mathrm{kg}$ groups and the 


\section{A}

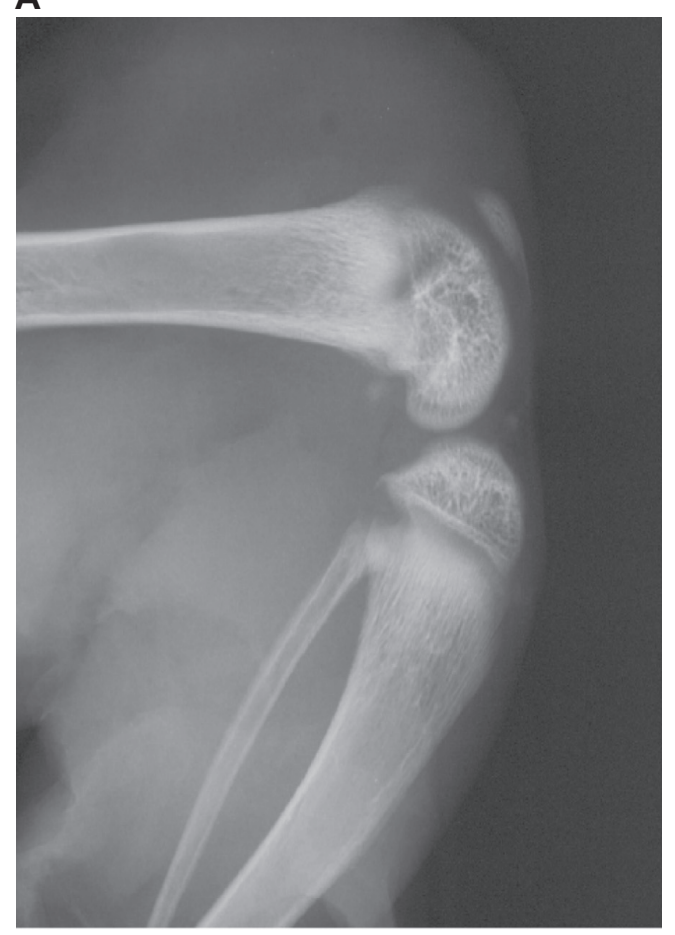

B

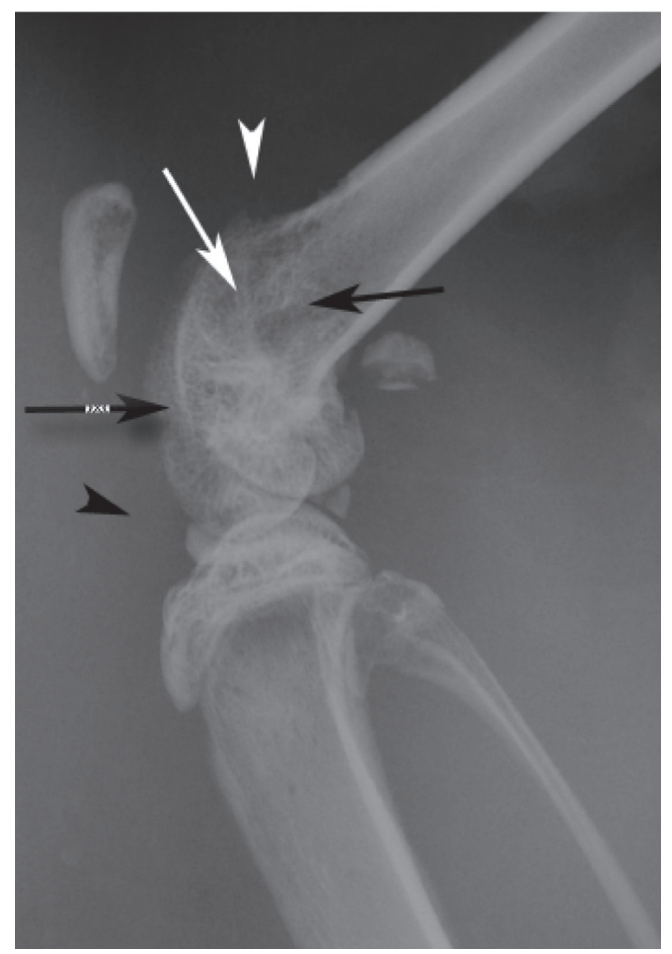

Figure 2 Radiographs of rat ipsilateral hind legs. Radiographs of the ipsilateral hind leg from vehicle (A) and MATLyLu (MLL) cell injected (B) rats displaying structural changes following model induction. Compared to vehicle, the radiograph from the MLL cell injected rat displayed acute osteopenia within the distal portion of the ipsilateral femur involving the distal femoral metadiaphysis (black arrow), metaphysis (white arrow), and epiphysis (black hatched arrow). Also visible in the radiograph of the MLL cell injected ipsilateral hind leg was cortical destruction in the anterior aspect of the metaphysis and metadiaphysis with periosteal reaction (white arrowhead). Joint effusion within the MLL cell-injected ipsilateral femur was also visible in the radiograph (black arrowhead).
A

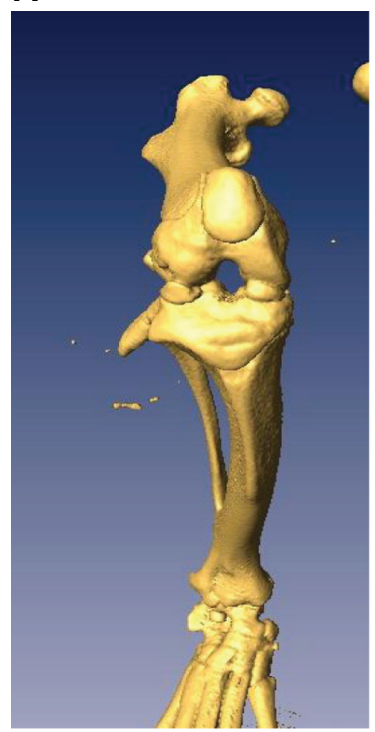

B

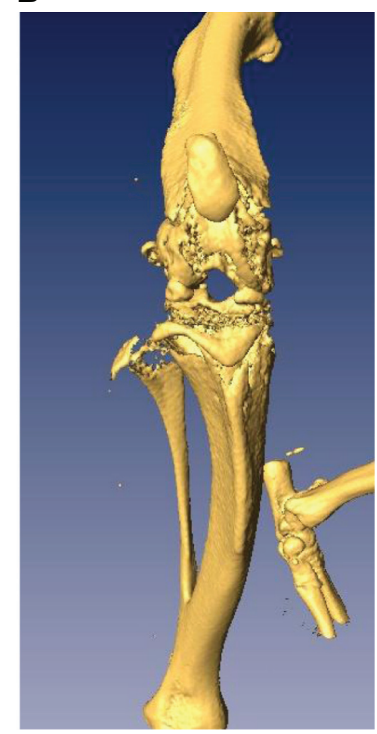

Figure 3 Computed tomography scans of rat ipsilateral hind legs. (A) Anterior view of the ipsilateral leg from a vehicle injected rat. (B) Anterior view of the ipsilateral leg from a MATLyLu (MLL) cell-injected rat. Structural damage in the form of trabecular and periosteal bone erosion is visible in the ipsilateral epiphysis from the MLL cell-injected rat with an absence of damage in the same region from the vehicle-injected rat.

vehicle group on days 10 and $13(P<0.001)$. The $5.0 \mathrm{mg} / \mathrm{kg}$ group was significantly different from the $1.0 \mathrm{mg} / \mathrm{kg}$ group on day $7(P<0.01)$. The $5.0 \mathrm{mg} / \mathrm{kg}$ group was significantly different from the PBS group on days 7, 10, and $13(P<0.001)$. The $2.5 \mathrm{mg} / \mathrm{kg}$ group was significantly different from the $1.0 \mathrm{mg} / \mathrm{kg}$ group on day $7(P<0.05)$. The $2.5 \mathrm{mg} / \mathrm{kg}$ group was significantly different from the PBS group on days 7 , 10 , and $13(P<0.001)$. The $1.0 \mathrm{mg} / \mathrm{kg}$ group was significantly different from the PBS group on days 7,10 , and 13 $(P<0.001)$. The vehicle group was significantly different

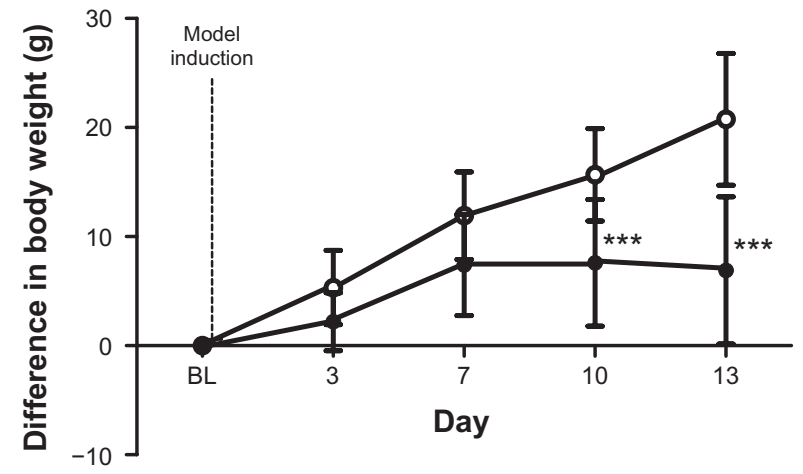

Figure 4 Difference in bodyweight following model induction. Change in bodyweight in vehicle $(n=12, \circ, 223.5 \mathrm{~g}$ at $B L)$ and $M L L$ cell $(n=10, \bullet, 228.4 g$ at $B L)$ rats following injection. Data are displayed as mean $\pm S D$. ***P $<0.001$.

Abbreviations: BL, baseline from reading from each group; MLL, MATLyLu; SD, standard deviation. 
A

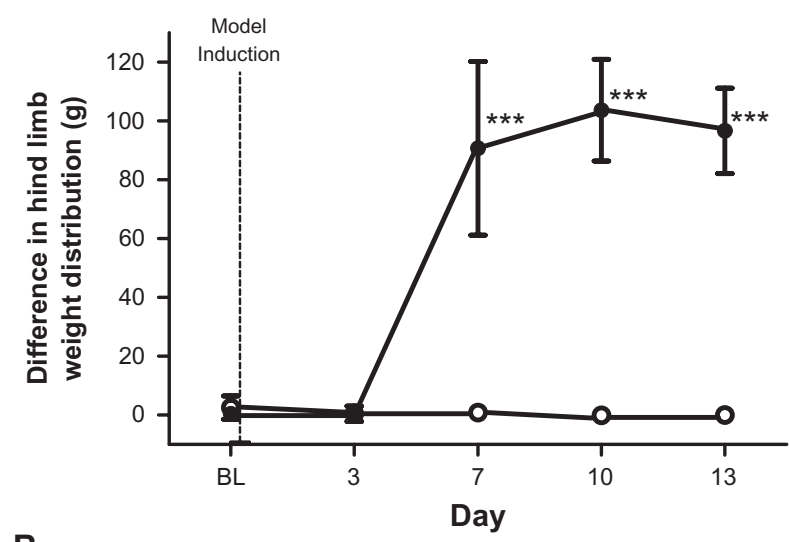

B

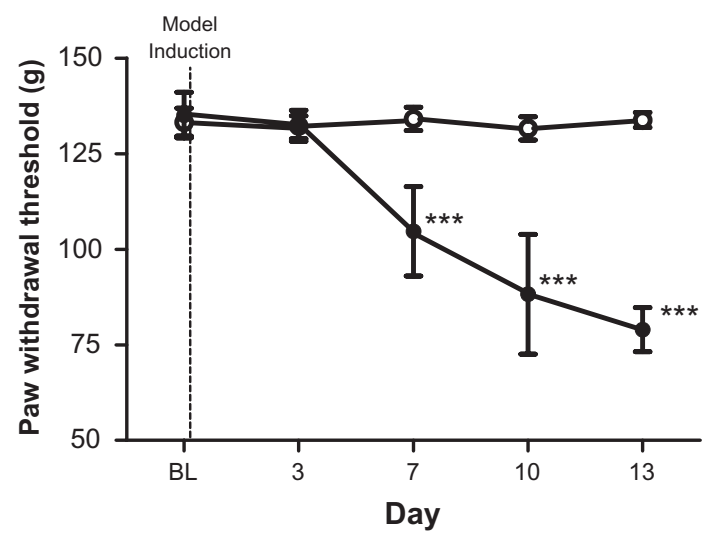

Figure 5 (A) Incapacitance test results. Figure displays the differential hind leg weight distribution in vehicle $(n=8, \circ)$ and $\operatorname{MLL}(n=10, \bullet)$ cell injected rats. Hind limb weight difference was measured in grams $(\mathrm{g})$. Data are displayed as mean $\pm \mathrm{SD}$. At $B L$, both groups displayed no significant difference in weight distribution between both hind legs. Dotted line indicates point of model induction. $* * * p<0.00$ I. (B) Randall-Selitto test results. Figure displays the paw withdrawal threshold in vehicle $(\mathrm{n}=8, \circ)$ and MLL $(\mathrm{n}=10, \bullet)$ cell-injected rats. Paw withdrawal threshold was measured in grams $(\mathrm{g})$ of force. Data are displayed as mean \pm SD. Dotted line indicates point of model induction. $* * * P<0.001$.

Abbreviations: BL, baseline from reading from each group; MLL, MATLyLu; SD, standard deviation.

from the PBS group on days 7,10 , and $13(P<0.001)$. The $5.0 \mathrm{mg} / \mathrm{kg}$ group reduced the difference in hind leg weight distribution when compared with the $2.5 \mathrm{mg} / \mathrm{kg}, 1.0 \mathrm{mg} / \mathrm{kg}$, vehicle, and PBS groups. Changes in weight difference in the $5.0 \mathrm{mg} / \mathrm{kg}$ group plateaus from day 7 to day 13 . Thus, the $5.0 \mathrm{mg} / \mathrm{kg}$ dose of meloxicam appears to be highly effective in reducing paw weight imbalance in the incapacitance test.

\section{Effect of meloxicam in the Randall-Selitto test}

Figure 6B illustrates the ipsilateral hind paw withdrawal threshold from the Randall-Selitto pressure clamp following different doses of meloxicam in the MLL cell-injected group and the group receiving PBS alone. The vehicle group
A
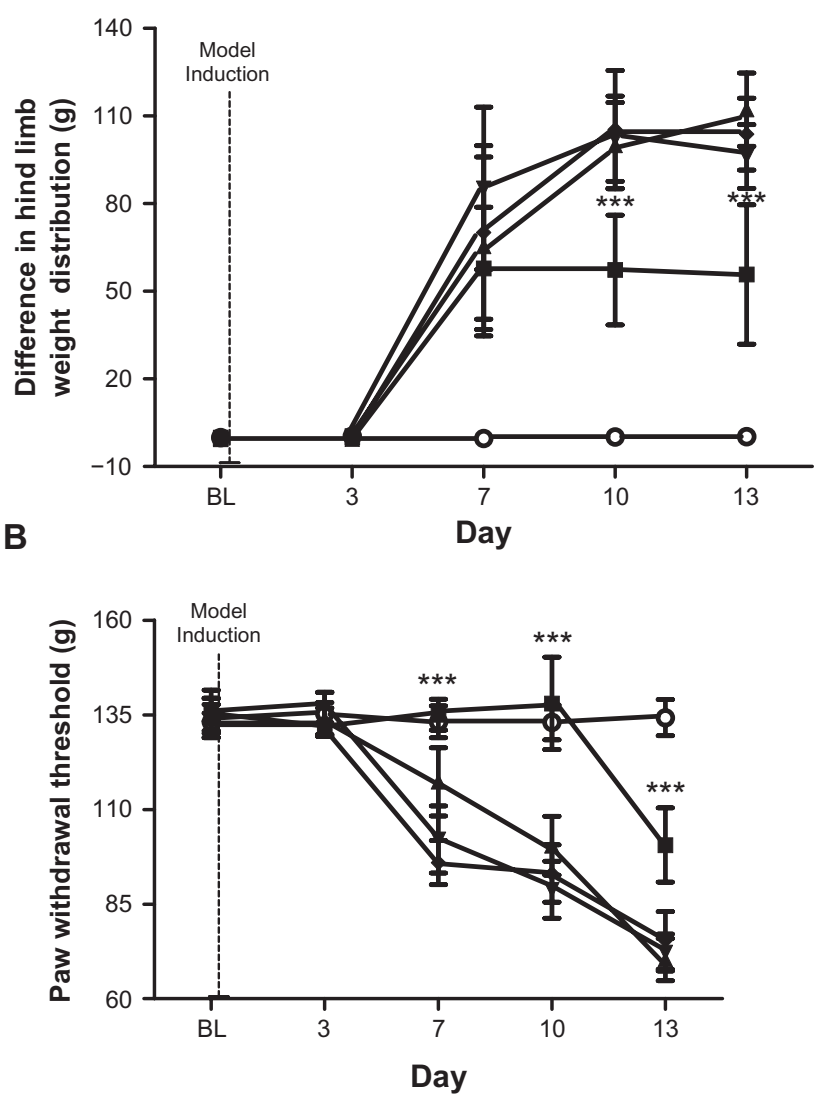

Figure 6 (A) Meloxicam effects in incapacitance test. Effect of meloxicam on differential hind leg weight distribution in PBS-injected (ie, no MLL cells), vehicle (ie, $0.5 \%$ methyl cellulose to MLL cell-injected rats) and in MLL cell-injected rats. PBS $(\circ, \mathrm{n}=8)$, vehicle $(\diamond, \mathrm{n}=9), 1.0 \mathrm{mg} / \mathrm{kg}$ meloxicam $(\boldsymbol{\nabla}, \mathrm{n}=9), 2.5 \mathrm{mg} / \mathrm{kg}$ meloxicam $(\boldsymbol{\Delta}, \mathrm{n}=8)$ and $5.0 \mathrm{mg} / \mathrm{kg}$ meloxicam $(\boldsymbol{n}, \mathrm{n}=7)$ groups are displayed. Data are displayed as mean $\pm S D$. $* * * P<0.001$. B) Meloxicam effects in Randall-Selitto test. Change in ipsilateral hind paw withdrawal threshold from the Randall-Selitto pressure clamp following administration of meloxicam or $0.5 \%$ methyl cellulose solution. Vehicle (०, $\mathrm{n}=8), \mathrm{PBS}(\star, \mathrm{n}=9), 1.0 \mathrm{mg} / \mathrm{kg}$ meloxicam $(\boldsymbol{\nabla}, \mathrm{n}=9), 2.5 \mathrm{mg} / \mathrm{kg}$ meloxicam $(\boldsymbol{\Lambda}, \mathrm{n}=8)$ and $5.0 \mathrm{mg} / \mathrm{kg}$ meloxicam $(\boldsymbol{m}, \mathrm{n}=7)$ groups are displayed. Data are displayed as $\pm \mathrm{SD}$. $* * * P<0.001$.

Abbreviations: BL, baseline from reading from each group; MLL, MATLyLu; PBS, phosphate buffered saline; SD, standard deviation.

received a $5 \%$ methyl cellulose solution. The $5.0 \mathrm{mg} / \mathrm{kg}$ group was significantly different from the $2.5 \mathrm{mg} / \mathrm{kg}, 1.0 \mathrm{mg} / \mathrm{kg}$, and vehicle groups on days 7,10 , and $13(P<0.001)$. The $5.0 \mathrm{mg} / \mathrm{kg}$ group was significantly different from the PBS group on day $13(P<0.001)$. The $2.5 \mathrm{mg} / \mathrm{kg}$ group was significantly different from the $1.0 \mathrm{mg} / \mathrm{kg}$ and vehicle groups on day $7(P<0.001)$. The $2.5 \mathrm{mg} / \mathrm{kg}$ group was significantly different from the $1.0 \mathrm{mg} / \mathrm{kg}$ group on day $10(P<0.001)$. The $2.5 \mathrm{mg} / \mathrm{kg}$ group was significantly different from the PBS group on days 7,10 , and $13(P<0.001)$. The $1.0 \mathrm{mg} / \mathrm{kg}$ group was significantly different from the PBS group on days 7,10 , and $13(P<0.001)$. The vehicle group was significantly different from the PBS group on days 7, 10, and 13 $(P<0.001)$. Rats receiving $5.0 \mathrm{mg} / \mathrm{kg}$ meloxicam maintained 
BL withdrawal threshold from the Randall-Selitto pressure clamp until day 10 of testing. Thus, it appears that $5.0 \mathrm{mg} / \mathrm{kg}$ of meloxicam was effective in delaying the decrease in withdrawal threshold.

\section{Discussion}

In this study, injection of MLL cells into the rat femoral epiphysis resulted in an aggressive osteolytic lesion. The resultant tumor formation led to trabecular bone degradation and the development of nociceptive behaviors.

Tumor growth as a result of MLL cell injection resulted in the appearance of nociceptive behaviors in injected rats. The incapacitance test demonstrated that rats receiving the MLL cell injection progressively favored the contralateral hind leg over the course of the experiment. Supporting this observation, Niiyama and colleagues recently demonstrated that injection of murine sarcoma cells NCTC 2472 into the mouse femur resulted in severely reduced weight bearing on the ipsilateral hind paw during standing. ${ }^{40}$ It has also been independently observed that intrafemoral injection of the NCTC 2472 murine sarcoma cells into rats resulted in the reduced weight-bearing ability on the ipsilateral hind leg. ${ }^{41}$ Medhurst and colleagues also demonstrated that following intra tibial injection of MRMT-1 rat mammary gland carcinoma cells the weight-bearing ability on the ipsilateral hind leg was reduced progressively over the course of the experiment. ${ }^{42}$ A similar progressive loss in weight-bearing ability in the ipsilateral hind leg following tumor formation was observed with intra femoral Walker-256 mammary gland cell injection. ${ }^{43}$

Moreover, tumor growth also reduced significantly the paw withdrawal threshold of the ipsilateral hind paw. Bone structure, growth of the tumor, and an inflammatory response are likely contributors to the observed nociceptive behaviors. The periosteum of the femur is richly innervated by primary afferent sensory nerve fibers. There are also peripheral nociceptive neurons located in the area of the patella and distal femur. The progressively growing tumor mass could have placed pressure upon the periosteal and peripheral nerve fibers resulting in their sensitization. Also, the presence of inflammatory factors leading to inflammation within the immediate area of tumor growth could have resulted in the sensitization of neurons. It has recently been demonstrated that the inflammatory mediator interleukin-1ß is increased in the area of an induced tibial osteosarcoma and within the inflamed paws of injected mice. ${ }^{44}$ Similar results have also been published by Zhang and colleagues. ${ }^{45}$

\section{Conclusion}

The findings from this project demonstrate that intra-femoral MLL cell injection leads to tumor formation and progressive bone destruction. The induced bone damage results in a progressive distribution of weight from the ipsilateral to the contralateral hind leg and a reduction in the ipsilateral paw withdrawal threshold. To our knowledge, this is the first study to provide a complete analysis of histological and radiographic results of rodent femurs combined with behavioral analysis in a model for CIBP induced by prostate cancer cell lines and via measurement of nociceptive scores. This model may be used in the future for therapeutic studies examining the pain associated with cancer induced bone metastasis.

\section{Disclosure}

The authors report no conflict of interest in this work.

\section{References}

1. Fidler IJ. Critical factors in the biology of human cancer metastasis: twenty-eighth G.H.A. Clowes memorial award lecture. Cancer Res. 1990; 50(19):6130-6138.

2. Guise TA, Kozlow WM, Heras-Herzig A, et al. Molecular mechanisms of breast cancer metastases to bone. Clin Breast Cancer. 2005;5 Suppl 2:S46-S53.

3. Mercadante S. Malignant bone pain: pathophysiology and treatment. Pain. 1997;69(1-2):1-18.

4. Miao XR, Gao XF, Wu JX, et al. Bilateral downregulation of Nav1.8 in dorsal root ganglia of rats with bone cancer pain induced by inoculation with Walker 256 breast tumor cells. BMC Cancer. 2010;10:216.

5. Saad F, Lipton A. Bone-marker levels in patients with prostate cancer: potential correlations with outcomes. Curr Opin Support Palliat Care. 2010;4(3):127-134.

6. Bagi CM. Targeting of therapeutic agents to bone to treat metastatic cancer. Adv Drug Deliv Rev. 2005;57(7):995-1010.

7. Landis SH, Murray T, Bolden S, Wingo PA. Cancer statistics, 1999. CA Cancer J Clin. 1999;49(1):1, 8-31.

8. Liu S, Yang J, Wang L, et al. Tibia tumor-induced cancer pain involves spinal p38 mitogen-activated protein kinase activation via TLR4dependent mechanisms. Brain Res. 2010;1346:213-223.

9. Coleman RE. Skeletal complications of malignancy. Cancer. 1997; 80 Suppl 8:1588-1594.

10. Coyle N, Adelhardt J, Foley KM, Portenoy RK. Character of terminal illness in the advanced cancer patient: pain and other symptoms during the last four weeks of life. J Pain Symptom Manage. 1990;5(2):83-93.

11. Fulfaro F, Casuccio A, Ticozzi C, Ripamonti C. The role of bisphosphonates in the treatment of painful metastatic bone disease: a review of phase III trials. Pain. 1998;78(3):157-169.

12. Lipton A. Bisphosphonates and breast carcinoma. Cancer. 1997; 80 Suppl 8:1668-1673.

13. Portenoy RK, Lesage P. Management of cancer pain. Lancet. 1999; 353(9165):1695-1700.

14. Rosier R. Bone pain. Am J Hosp Palliat Care. 1992;9(6):37.

15. Tanko LB, Karsdal MA, Christiansen C, Leeming DJ. Biochemical approach to the detection and monitoring of metastatic bone disease: what do we know and what questions need answers? Cancer Metastasis Rev. 2006;25(4):659-668.

16. Geis C, Graulich M, Wissmann A, et al. Evoked pain behavior and spinal glia activation is dependent on tumor necrosis factor receptor 1 and 2 in a mouse model of bone cancer pain. Neuroscience. 2010;169(1): 463-474. 
17. Hald A, Hansen RR, Thomsen MW, et al. Cancer-induced bone loss and associated pain-related behavior is reduced by risedronate but not its phosphonocarboxylate analog NE-10790. Int J Cancer. 2009;125(5): 1177-1185.

18. Mantyh PW, Clohisy DR, Koltzenburg M, Hunt SP. Molecular mechanisms of cancer pain. Nat Rev Cancer. 2002;2(3):201-209.

19. Pacharinsak C, Beitz A. Animal models of cancer pain. Comp Med. 2008;58(3):220-233

20. Mercadante S, Arcuri E. Breakthrough pain in cancer patients: pathophysiology and treatment. Cancer Treat Rev. 1998;24(6):425-432.

21. Portenoy RK, Hagen NA. Breakthrough pain: definition, prevalence and characteristics. Pain. 1990;41(3):273-281.

22. Portenoy RK, Payne D, Jacobsen P. Breakthrough pain: characteristics and impact in patients with cancer pain. Pain. 1999;81(1-2):129-134.

23. Clohisy DR, Mantyh PW. Bone cancer pain. Clin Orthop Relat Res. 2003;415 Suppl:S279-S288.

24. Mercadante $\mathrm{S}$. Controversies over spinal treatment in advanced cancer patients. Support Care Cancer. 1998;6(6):495-502.

25. Portenoy RK. Managing cancer pain poorly responsive to systemic opioid therapy. Oncology (Williston Park). 1999;13(5 Suppl 2):25-29.

26. Smolev JK, Heston WDW, Scott WW, et al. Characterization of the Dunning R-3327-H prostatic carcinoma: an appropriate animal model for prostatic cancer. Can Treat Rep. 1977;61:273.

27. Issacs JT, Yu GW, Coffey DS. The characterization of a newly identified, highly metastatic variety of Dunning R3327 rat prostatic adenocarcinoma system: the MATLyLu tumor. Invest Urol. 1981;19(1): 20-23.

28. Lubaroff DM, Canfield L, Feldbush TL, et al. R3327 adenocarcinoma of the copenhagen rat as a model for the study of the immunologic aspects of prostate cancer. J Natl Cancer Inst. 1977;58(6):1677-1689.

29. Isaacs JT, Heston WDW, Weismann RM, et al. Animal models of hormone sensitive and insensitive prostatic adenocarcinomas: Dunning R-3327-H, R-3327-HI and R-3327-AT. Cancer Res. 1978;38(11): 4353-4359.

30. Issacs JT, Issacs WB, Coffey DS. Models for the development of nonreceptor methods for distinguishing androgen sensitive and insensitive prostatic tumors. Cancer Res. 1979;39:2652.

31. Wenger AS, Mickey DD, Hall M, et al. In vitro characterization of MATLyLu: a Dunning rat prostate adenocarcinoma tumor subline. J Urol. 1984;131:1232-1236.

32. Noble S, Balfour JA. Meloxicam. Drugs. 1996;51(3):424-430.
33. Fleischmann R, Imran I, Slobodin G. Meloxicam. Expert Opin Pharmacother. 2002;3(10):1501-1512.

34. Engelhardt G, Bögel R, Schnitzer C, et al. Meloxicam: influence on arachidonic acid metabolism. Part I. In vitro findings. Biochem Pharmacol. 1996;51(1):21-28.

35. Naruse T, Nishida Y, Hosono K, et al. Meloxicam inhibits osteosarcoma growth, invasiveness and metastasis by COX-2-dependent and independent routes. Carcinogenesis. 2006;27(3):584-592.

36. Pairet M, Engelhardt G. The preferential inhibition of COX-2 by meloxicam is hightly dependent on the structure of the drug. Eur J Rheumatol Inflamm. 1995;24 Supp1 3:272.

37. Engelhardt G. Pharmacology of meloxicam, a new non-steroidal antiinflammatory drug with an improved safety profile through preferential inhibition of COX-2. Br J Rheumatol. 1996;35 Suppl 1:4-12.

38. Goldman AP, Williams CS, Sheng H, Lamps LW, et al. Meloxicam inhibits the growth of colorectal cancer. Carcinogenesis. 1998;19: 2195-2199.

39. Tsubouchi Y, Mukai S, Kawahito Y, Yamada R, et al. Meloxicam inhibits the growth of non-small cell lung cancer. Anticancer Res. 2000; 20:2867-2872.

40. Niiyama Y, Kawamata T, Yamamoto J, et al. SB366971, a TRPV1 antagonist, potentiates analgesic effects of systemic morphine in a murine model of bone cancer pain. Br J Anaesth. 2009;102(2):251-258.

41. Hald A, Ding M, Egerod K, et al. Differential effects of repeated low dose treatment with the cannabinoid agonist WIN 55,212-2 in experimental models of bone cancer pain and neuropathic pain. Pharmacol Biochem Behav. 2008;91(1):38-46.

42. Medhurst SJ, Walker K, Bowes M, et al. A rat model of bone cancer pain. Pain. 2002;96(1-2):129-140.

43. Mao-Ying QL, Zhao J, Dong ZQ, et al. A rat model of bone cancer pain induced by intra-tibia inoculation of Walker 256 mammary gland carcinoma cells. Biochem Biophys Res Commun. 2006;345(4): 1292-1298.

44. Baamonde A, Curto-Reyes V, Juarez L, et al. Antihyperalgesic effects induce by the IL-1 receptor antagonist anakinra and increased IL-1beta levels in inflamed and osteosarcoma-bearing mice. Life Sci. 2007; 81(8):673-682.

45. Zhang RX, Liu B, Li A, et al. Interleukin 1beta facilitates bone cancer pain in rats by enhancing NMDA receptor NR-1 subunit phosphorylation. Neuroscience. 2008;154(4):1533-1538.
Journal of Pain Research

\section{Publish your work in this journal}

The Journal of Pain Research is an international, peer-reviewed, open access, online journal that welcomes laboratory and clinical findings in the fields of pain research and the prevention and management of pain. Original research, reviews, symposium reports, hypothesis formation and commentaries are all considered for publication.

\section{Dovepress}

The manuscript management system is completely online and includes a very quick and fair peer-review system, which is all easy to use. Visit http://www.dovepress.com/testimonials.php to read real quotes from published authors. 